\title{
Discussion on the Harm of Antibiotics to Human Health in Water Environment and Its Countermeasures
}

\author{
Yu Wang \\ Zhuhai College of Jilin University \\ Zhuhai, China
}

\begin{abstract}
The development and application of antibiotics have changed the lives of human beings and contributed important strengths to human health. However, with the increasing abuse of antibiotics, the harm caused by antibiotics to human sustainable development is becoming more and more obvious. Antibiotics are made due to their powerful application functions. It is not only used in human life, but also widely used in aquaculture and livestock breeding. Excessive antibiotic application and residue make natural water bodies also contaminated by antibiotics. Through exploring the harm in water environment of antibiotics to human health and its countermeasures, this paper analyzes the main pollution sources of antibiotics in water environment and the harm to human health caused by antibiotics pollution in water environment and puts forward feasible countermeasures to promote the rational application of antibiotics and ensure sustainable development of human health.
\end{abstract}

Keywords-water environment; antibiotics; human health; hazard; countermeasures

\section{INTRODUCTION}

Antibiotics are mainly applied to humans or animals. After the drugs are metabolized by humans or animals, excessive antibiotics will enter the natural environment in the form of original forms, causing pollution of the natural water environment. The water environment is a circulatory system of the natural environment. Antibiotics in the water environment will follow the circulation system of the water to carry out recycling application of biological chain. Although antibiotics can exert great medical effects on disease treatment, the pollution of water environment will cause great side effects for human health. Analysis of hazards is of great significance to the healthy development of human beings.

\section{POLLUTION SOURCE OF ANTIBIOTICS IN WATER}

\section{A. Medical Antibiotic Contamination}

Medical antibiotics have been identified as one of the main sources of antibiotic contamination. China is one of the countries with the largest number of medical antibiotics uses. Eighty percent of the patients who enter the hospital for treatment are treated with antibiotics, and the virus is constantly evolving. In response, the application of a single antibiotic has gradually evolved into a combination of two or more antibiotics. Moreover, only a small amount of antibiotics enters the human body, and the rest of them will be metabolized by the human body, and most antibiotics will be excreted in the original active form through excretion. The discharge will enter the sewage discharge system, but the system cannot degrade these active antibiotics. Then, it will follow the purified water into the surface water body, and some water bodies can naturally penetrate into the groundwater body.

\section{B. Animal Antibiotic Contamination}

The pollution of animal antibiotics mainly refers to the pollution caused by the use of antibiotics in the process of animal husbandry and aquaculture and management. With the increase of national economic income, the material life of the people is gradually enriched, which brings great development space to the development of animal husbandry and aquaculture industry. In order to improve the production rate of animal products and aquaculture products to increase the income, the abuse of antibiotics is more common, and even the farmers have used antibiotics as conventional drugs to prevent animal diseases, so that the retention of antibiotics in animal products and aquatic products is very common, thus forming " the circular mode of animal husbandry and aquaculture productshuman- excrement -natural water, leading to antibiotic pollution in water bodies. In the process of animal husbandry, there is the other important way of pollution is "animal excrement - natural water body". When many farmers are carrying out animal husbandry, the excreta are basically produced by natural discharge, which will be accompanied by the scour of rainwater and flow into the natural surface water body or penetrate into underground water, resulting in antibiotic pollution in natural water environment. In the breeding process of aquatic products, some farmers will put antibiotics into the aquaculture water in order to prevent and cure the disease, and because of the influence of the industry factors of aquaculture, the excessive use of antibiotics has a long history in its development, and what's worse, the industry has already produced strong dependence on antibiotics. The existence of these problems will form direct pollution to the natural water body. In the course of natural circulation, it will cause damage to other water resources and cause the pollution of water environment. 


\section{Antibiotic Contamination from Industrial Wastewater}

In this paper, the industrial wastewater mainly refers to the industrial wastewater produced by pharmaceutical companies in the production process. In the process of antibiotic production, a large amount of industrial wastewater is generated, including intermediate chemical products in the production process of antibiotics and direct antibiotic products. China has relatively strict emission standards for wastewater discharge of pharmaceutical plants. However, in actual work, many regions will reduce the wastewater discharge standards of pharmaceutical companies in order to increase investment or use taxation of pharmaceutical companies to increase local income. Even some untreated industrial wastewater will be directly discharged in some companies, but the local regulatory authorities have turned a deaf ear. The excessive discharge of these industrial wastewaters has caused huge pollution to the water environment.

\section{ANALYSIS OF THE HARM OF ANTIBIOTICS TO HUMAN HEALTH IN WATER ENVIRONMENT}

\section{A. Production of Antibiotic Residues in Agricultural and Sideline Products, Affecting Human Diet Safety}

The antibiotic residues in animal husbandry products and aquatic products can cause direct drug damage to the human body. Long term use of antibiotics as the prevention and control measures of the epidemic disease will cause a large number of antibiotic residues in the animal body. When the products are eaten by human beings, these substances will be taken into the human body, and the human body is in the human body. With the disease, the use of antibiotics can be used to treat the disease, but when there is no disease in the human body, a long period of concentration of drugs will cause strong resistance to the body, and then the function of the body's own immune system will be reduced. At present, in the field of food, pork, beef, poultry meat products, aquatic products and dairy products have been detected the existence of antibiotic residues in different degrees. The unsafe food chain will cause huge hidden dangers to mankind in the future.

\section{B. Antibiotic Pollution Will Aggravate the Production of Human Drug-resistant Strains}

The improper use of antibiotics in various forms will cause resistance to animals or human bodies. The bacterial drug resistance in the water environment is also the focus of the world health community. The bacteria in the water will inherit and transfer the drug-resistant genes through the way of gene change. These drug-resistant bacteria will be brought into the human body through some contacts, such as drinking water or swimming., so that the intestinal bacteria of the human body are also resistant to drug. The production of drug-resistant bacteria will bring the malignant result of non-drug treatment to human health. At present, the existence of superbacteria has been detected in China. Superbacteria have strong resistance to broad-spectrum antibiotics, which not only cause damage to human life and property, but also cause serious social crisis.

\section{Causing Severe Allergic Reactions and Side Effects for Human}

Although the concentration of antibiotics in the water environment is not high and it will not directly cause death, its far-reaching impact cannot be ignored. Some patients will have acute allergic reactions due to the use of antibiotics, and some patients will suffer the gradually increased burden of liver and kidney function due to accumulation of antibiotics in the body, thus causing some induced diseases. Its damage to organs is irreversible. The toxic side effects of antibiotics in the water environment are a long-term process. Especially for people with sensitive physiques such as pregnant women, the enrichment of antibiotics may even affect the fetus health.

\section{Increasing Treatment Cycle and Financial Burden of Patients}

In the course of clinical medical treatment in China, bacteria such as Escherichia coli, staphylococcus aureus and Klebsiella pneumoniae have been resistant to drugs to a certain extent. The existence of these bacteria will bring great impact on the development of our medical service in the future and will also cause great damage to human health. The fundamental reason is the current abuses of antibiotics. The resistance of these basic pathogens will result in a disease that would otherwise achieve a desired therapeutic effect with a single or one-cycle antibiotic treatment requires a combination of multiple antibiotics and multiple cycles of treatment to achieve its therapeutic goals. It has caused a vicious cycle pattern of excessive use of antibiotics, and the extension of the treatment cycle has undoubtedly increased the patient's physical burden and economic burden.

\section{COUNTERMEASURES FOR PREVENTING AND CONTROLLING ANTIBIOTIC CONTAMINATION IN WATER ENVIRONMENT}

\section{A. Strengthening Supervision of the Production and Circulation of Antibiotics}

It's important to strengthen the supervision of antibiotic production field, forcing antibiotic production enterprises to strictly follow the related production standards to carry out drug production; strengthen the effective supervision of the sewage discharge, strictly prohibiting the untreated production sewage discharge; strengthen the renovation of the illegal sewage enterprises, so that the related production enterprises can enhance their sense of responsibility and cannot and do not dare to conduct illegal sewage disposal. At the same time, the supervision of the circulation of antibiotics should be tightened to ensure that the circulation of antibiotics can enter the regular medical and health places, and the drugs can be guided by professional medical and health personnel for scientific use.

\section{B. Improving the Professional Quality of Medical Workers and Carrying out Rational Use of Antibiotics}

Medical staff should have a systematic knowledge of the antibiotics application and be able to formulate the best treatment plan. Clinicians should establish their own scientific use concept in the treatment of patients with antibiotics. The 
use classification of antibiotics should be carried out strictly in accordance with the antibacterial spectrum and the use dose should be strictly in accordance with the relevant medical operating standards. Based on the specific conditions of the disease, it's crucial to establish a comprehensive treatment plan, and combined medication or joint antibiotics use can be used for drug treatment. They are also required to perform regular effect examination and can do accurately assess the development of the disease and give changes to the appropriate treatment plan to ensure the best treatment. At the same time, clinicians should actively communicate with patients and their families to guide them to master the correct use of antibiotics, so that they can realize the importance of scientific drugs and be able to use scientific and rational drugs in accordance with relevant regulations.

\section{Strengthening the Rational Use of Antibiotics in the Outpatient Service}

The prescription medication of the outpatient is also an important part of the irrational use of antibiotics. After the use of the drug, the patient cannot be monitored for adverse drug performance and treatment, so that the dosage of the drug cannot be effectively analyzed objectively, which demands the relevant departments should establish the whole system of antibiotic drug use in the clinic diagnosis and treatment link. The doctor needs to make strict regulations on the way of drug use, the time of drug use and the dosage of the drug and pay attention to the history of the patient's drug use to make sure the accurate use of antibiotics in the outpatient department.

\section{Strictly Controlling the Use of Antibiotics in Animal Husbandry and Aquaculture Industry}

Because the livestock breeding and aquaculture industry are the areas of overuse of antibiotics, the relevant units need to strengthen the propaganda of the application of antibiotics these two fields so that the farmers can recognize that the improper use of antibiotics can cause severe water pollution and human development. Through the strengthening of their personal knowledge, they can consciously abide by the use principle of additives and veterinary drugs, making reasonable use of antibiotics in culture industry. At the same time, the development of pollution-free medicaments and the substitute for antibiotic feed additives will be intensified so that the farmers can achieve a win-win situation of their interest protection and environmental protection, thus promoting the sustainable development of animal husbandry and aquaculture industry and ensuring the safety of human diet.

\section{CONCLUSION}

At present, China's medical and health industry and the pharmaceutical industry are developing at a relatively fast rate. The medical industry and the public have made the overuse of antibiotics in the medical industry more serious in order to achieve better therapeutic effects. The breeding industry has also ensured the realization of its own economic interests by excessive use of antibiotics, but at the same time, China's sewage treatment technology is still relatively lagging, and the antibiotic pollution in the water environment cannot be effectively solved. The water environment will cause the antibiotics to be rich in the human body due to the natural circulation of water, which in turn causes the decline of human immunity and the emergence of drug-resistant beads. The long-term harm of the accumulation of antibiotics to human health remains to be studied. Yet, it is undeniable that it will cause very serious hidden dangers to human health. The relevant departments must guide the rational use of antibiotics in the field of health care and culture, thereby reducing the discharge of antibiotic waste, reducing the pollution and recycling use of natural waters, and avoiding the great threat to human health as far as possible.

\section{REFERENCES}

11] Qian Zhuozhen, Luo Donglian, Luo Fangfang. Determination of Peptide Antibiotic Residues in Cultured Sediments by High Performance Liquid Chromatography-Tandem Mass Spectrometry[J]. Analytical Chemistry,2016,44(6):870-875.

[2] Chu Weibin, Shi Binlin, Hong Lei. Application, Harms and Scientific Use of Antibiotics in Livestock and Poultry Production[J]. Journal of Anhui Agricultural Sciences,2015, (19):128-130.

[3] Zhang Bowen, Lang Lang. Study on Antibiotic Pollution and Toxicity in Water Environment[J]. Heilongjiang Science and Technology Information,2016,(33):128-129. 